\title{
New physics upper bound on the branching ratio of $B_{s} \rightarrow l^{+} l^{-}$and $B_{s} \rightarrow l^{+} l^{-} \gamma$.
}

\author{
Ashutosh Kumar Alok ${ }^{a}$ and S. Uma Sankar ${ }^{a}$ \\ ${ }^{a}$ Department of Physics, Indian Institute of Technology, Bombay, \\ Mumbai 400076, India
}

We consider the most general new physics effective Lagrangian for $b \rightarrow s l^{+} l^{-}$. We derive the upper limit on the branching ratio for the processes $B_{s} \rightarrow l^{+} l^{-}$where $l=e, \mu$, subject to the current experimental bounds on related processes, $B \rightarrow\left(K, K^{*}\right) l^{+} l^{-}$. If the new physics interactions are of vector/axial-vector form, the present measured rates for $B \rightarrow\left(K, K^{*}\right) l^{+} l^{-}$constrain $B\left(B_{s} \rightarrow l^{+} l^{-}\right)$to be of the same order of magnitude as their respective Standard Model (SM) predictions. On the other hand, if the new physics interactions are of scalar/pseudoscalar form, $B \rightarrow\left(K, K^{*}\right) l^{+} l^{-}$rates do not impose any useful constraint on $B\left(B_{s} \rightarrow l^{+} l^{-}\right)$and the branching ratios of these decays can be as large as present experimental upper bounds. If future experiments measure $B\left(B_{s} \rightarrow l^{+} l^{-}\right)$ to be $\geq 10^{-8}$ then the new physics giving rise to these decays has to be of the scalar/pseudoscalar form. We also consider the effect of new physics on $B\left(B_{s} \rightarrow l^{+} l^{-} \gamma\right)$ subject to the present experimental constraints on $B \rightarrow\left(K, K^{*}\right) l^{+} l^{-}$and $B \rightarrow K^{*} \gamma$. New physics in form scalar/pseudoscalar, which makes a very large contribution to $B_{s} \rightarrow l^{+} l^{-}$, makes no contribution at all to $B_{s} \rightarrow l^{+} l^{-} \gamma$ due to angular momentum conservation. New Physics in the form of vector/axial-vector operators is constrained by the data on $B \rightarrow\left(K, K^{*}\right) l^{+} l^{-}$and new physics in the form of tensor/pseudo-tensor is constrained by the data on $B \rightarrow K^{*} \gamma$. In both cases, enhancement of $B\left(B_{s} \rightarrow l^{+} l^{-} \gamma\right)$ much beyond the SM expectation is impossible. In conclusion, present data on $B \rightarrow\left(K, K^{*}\right)$ transitions allow for large $B\left(B_{s} \rightarrow l^{+} l^{-}\right)$but do not allow $B\left(B_{s} \rightarrow l^{+} l^{-} \gamma\right)$ to be much larger than its SM expectation.

\section{INTRODUCTION}

The rare decays of $B$ mesons involving flavour changing neutral interactions (FCNI) $b \rightarrow s$ have been a topic of great interest for long. Not only will they subject the Standard Model (SM) to accurate tests but will also put strong constaraints on several models beyond the SM. Recently, the very high statistics experiments at B-factories have measured non-zero values for the branching ratios for the FCNI processes $B \rightarrow\left(K, K^{*}\right) l^{+} l^{-}$ [12],

$$
\begin{aligned}
B\left(B \rightarrow K l^{+} l^{-}\right) & =\left(4.8_{-0.9}^{+1.0} \pm 0.3 \pm 0.1\right) \times 10^{-7} \\
B\left(B \rightarrow K^{*} l^{+} l^{-}\right) & =\left(11.5_{-2.4}^{+2.6} \pm 0.8 \pm 0.2\right) \times 10^{-7}
\end{aligned}
$$

These branching ratios are close to the values predicted by the SM [3. However, the SM predictions for them contain about $\sim 15 \%$ uncertainty coming from the hadronic form factors. Still, it is worth considering what constraints these measurements impose on other related processes.

In section 2 and 3 we will discuss the impact of there measurements on the predictions for $B_{N P}\left(B_{s} \rightarrow l^{+} l^{-}\right)$and $B_{N P}\left(B_{s} \rightarrow l^{+} l^{-} \gamma\right)$ respectively [4].

\section{NEW PHYSICS UPPER BOUND ON $B\left(B_{s} \rightarrow l^{+} l^{-}\right)$.}

The same $b \rightarrow s l^{+} l^{-}$four Fermi interaction is responsible for both leptonic decays $B_{s} \rightarrow l^{+} l^{-}$ and semi-leptonic decays $B \rightarrow\left(K, K^{*}\right) l^{+} l^{-}$. The SM predictions for the branching ratios for the decays $B_{s} \rightarrow e^{+} e^{-}$and $B_{s} \rightarrow \mu^{+} \mu^{-}$are $(7.58 \pm$ $3.5) \times 10^{-14}$ and $(3.2 \pm 1.5) \times 10^{-9}$ respectively [6]. The large uncertainy in the SM prediction for these branching ratios arises due to the $12 \%$ uncertainty in the $B_{s}$ decay constant and $10 \%$ uncertainty in the measurement of $V_{t s}$. 
$B_{s} \rightarrow l^{+} l^{-}$has been studied in various models, both with and without natural flavour conservation, before. In both these kinds of models it was shown that $B_{s} \rightarrow \mu^{+} \mu^{-}$can have a branching raio of $\geq 10^{-8} \quad 78$. From the experimental side, at present, there exist only the upper bound $B\left(B_{s} \rightarrow \mu^{+} \mu^{-}\right)<1.0 \times 10^{-7}$ at $95 \%$ C.L. 9].

The effective new physics Lagrangian for $b \rightarrow$ $s l^{+} l^{-}$transitions can be written as,

$L_{e f f}\left(b \rightarrow s l^{+} l^{-}\right)=L_{V A}+L_{S P}+L_{T}$.

where, $L_{V A}$ contains vector and axial-vector couplings, $L_{S P}$ contains scalar and psuedo-scalar couplings and $L_{T}$ contains tensor couplings. $L_{T}$ does not contribute to $B_{s} \rightarrow l^{+} l^{-}$because $\left\langle 0\left|\bar{s} \sigma^{\mu \nu} b\right| B_{s}\left(p_{B}\right)\right\rangle=0$. Hence we will drop it from further consideration. We consider $L_{V A}$ and $L_{S P}$ one at a time.

We parametrize $L_{V A}$ as,

$$
\begin{aligned}
L_{V A}\left(b \rightarrow s l^{+} l^{-}\right)= & \frac{G_{F}}{\sqrt{2}}\left(\frac{\alpha}{4 \pi s_{W}^{2}}\right) \bar{s}\left(g_{V}+g_{A} \gamma_{5}\right) \gamma_{\mu} b \\
& \bar{l}\left(g_{V}^{\prime}+g_{A}^{\prime} \gamma_{5}\right) \gamma^{\mu} l
\end{aligned}
$$

Here the constants $g$ and $g^{\prime}$ are the effective couplings which characterise the new physics. The calculation of decay rate gives,

$$
\begin{aligned}
\Gamma_{N P}\left(B_{s} \rightarrow l^{+} l^{-}\right)= & \frac{G_{F}^{2} f_{B_{s}}^{2}}{8 \pi}\left(\frac{\alpha}{4 \pi s_{W}^{2}}\right)^{2} \\
& \left(g_{A} g_{A}^{\prime}\right)^{2} m_{B_{s}} m_{l}^{2} .
\end{aligned}
$$

Thus the decay rate depends upon the value of $\left(g_{A} g_{A}^{\prime}\right)^{2}$. To estimate the value of $\left(g_{A} g_{A}^{\prime}\right)^{2}$, we look at semi-leptonic decays. We first consider $B \rightarrow K^{*} l^{+} l^{-}$. The decay rate is,

$$
\begin{aligned}
\Gamma_{N P}\left(B \rightarrow K^{*} l^{+} l^{-}\right)= & \frac{1}{2}\left(\frac{G_{F}^{2} m_{B}^{5}}{192 \pi^{3}}\right)\left(\frac{\alpha}{4 \pi s_{W}^{2}}\right)^{2} \\
& \left(g_{V}^{\prime 2}+g_{A}^{\prime 2}\right) I_{V A}
\end{aligned}
$$

where $I_{V A}=g_{V}^{2} V^{2} I_{1}+g_{A}^{2} A_{1}^{2} I_{2} . \quad I_{1}$ and $I_{2}$ are integrals over the dilepton invariant mass $(z=$ $\left.q^{2} / m_{B}^{2}\right)$.

$\Gamma_{N P}\left(B \rightarrow K^{*} l^{+} l^{-}\right)$depends on both vector and axial vector couplings. To get a handle on vector couplings we look at $B \rightarrow K l^{+} l^{-}$. The decay rate is given by,

$$
\begin{aligned}
\Gamma_{N P}\left(B \rightarrow K l^{+} l^{-}\right)= & \left(\frac{G_{F}^{2} m_{B}^{5}}{192 \pi^{3}}\right)\left(\frac{\alpha}{4 \pi s_{W}^{2}}\right)^{2} \\
& g_{V}^{2}\left(g_{V}^{\prime 2}+g_{A}^{\prime 2}\right)\left(\frac{f^{+}(0)}{2}\right)^{2} .
\end{aligned}
$$

We are trying to see what is the maximum value of $\left(g_{A} g_{A}^{\prime}\right)^{2}$, consistent with semi-leptonic data. To get this, we make the approximation $\Gamma_{\exp }=$ $\Gamma_{N P}$, i.e. the experimentally measuted semileptonic branching ratios are saturated by new physics couplings. Under this approximation, we get

$g_{A}^{2}\left(g_{V}^{\prime 2}+g_{A}^{\prime 2}\right)=\left(6.76_{-3.48}^{+4.04}\right) \times 10^{-3}$.

Here all the errors were added in quadrature and the values of form-factors were taken from 10.

Therefore the upper bounds on the branching ratios are $B\left(B_{s} \rightarrow e^{+} e^{-}\right)<1.20 \times$ $10^{-13}$ and $B\left(B_{s} \rightarrow \mu^{+} \mu^{-}\right)<5.13 \times 10^{-9}$ at $3 \sigma$. These bounds are similar to SM predictions. It should not be surprising because $\Gamma=$ $(\text { c.c. })^{2}(f . f .)^{2}$ phase space. In semi-leptonic case $\Gamma_{e x p}=\Gamma_{S M}$. Then we assumed $\Gamma_{N P}=\Gamma_{e x p}$ which implies $(c . c)_{N P}=(c . c)_{S M}$ and hence $\Gamma_{N P}\left(B_{s} \rightarrow l^{+} l^{-}\right)=\Gamma_{S M}\left(B_{s} \rightarrow l^{+} l^{-}\right)$. A more stringent upper bound is obtained if we equate the new physics branching ratio to the difference between the expeimental value and the SM prediction. Therefore, given the measured values of branching ratios of $B \rightarrow\left(K, K^{*}\right) l^{+} l^{-}$by Belle and BaBar, new physics cannot boost $B_{s} \rightarrow l^{+} l^{-}$ above $S M$ value if it is of the form vector/axialvector.

Let us turn now to $L_{S P}$ with scalar and pseudoscalar couplings.

$$
\begin{aligned}
L_{S P}\left(b \rightarrow s l^{+} l^{-}\right)= & \frac{G_{F}}{\sqrt{2}}\left(\frac{\alpha}{4 \pi s_{W}^{2}}\right) \bar{s}\left(g_{S}+g_{P} \gamma_{5}\right) b \\
& \bar{l}\left(g_{S}^{\prime}+g_{P}^{\prime} \gamma_{5}\right) l .
\end{aligned}
$$

The Branching ratio is given by,

$B\left(B_{s} \rightarrow l^{+} l^{-}\right)=0.17 \frac{f_{B_{s}}^{2} g_{P}^{2}\left(g_{S}^{\prime 2}+g_{P}^{\prime 2}\right)}{\left(m_{b}+m_{s}\right)^{2}}$.

To get a bound on $g_{P}^{2}\left(g_{S}^{\prime 2}+g_{P}^{\prime 2}\right)$ we need to consider only $B \rightarrow K^{*} l^{+} l^{-}$. Here again we make the 
approximation $\Gamma_{\text {exp }}=\Gamma_{N P}$. Under this approximation we get,

$g_{P}^{2}\left(g_{S}^{\prime 2}+g_{P}^{\prime 2}\right)=\frac{\left(m_{b}-m_{s}\right)^{2} B_{E x p}\left(B \rightarrow K^{*} l^{+} l^{-}\right)}{2.16\left[A_{0}(0)\right]^{2} \times 10^{-3}}(10)$

Substituting this in $\left(B_{s} \rightarrow l^{+} l^{-}\right)$rate we get,

$B\left(B_{s} \rightarrow \mu^{+} \mu^{-}\right)=(2 \pm 1) \times 10^{-5}$.

The upper bound on $B\left(B_{s} \rightarrow \mu^{+} \mu^{-}\right)$from the above equation is much higher than the present experimental upper bound 9]. Thus we see that if new phsyics effective Lagrangian is of the scalar/pseudoscalar form, then the present measurements of semi-leptonic rates DO NOT provide any useful constraints on $B_{s} \rightarrow l^{+} l^{-}$. Therefore if experiments at Tevatron or LHCb find that $B\left(B_{s} \rightarrow \mu^{+} \mu^{-}\right) \geq 10^{-8}$, then we can immediately conclude that the new phsyics responsible for it is of scalar/pseudoscalar type.

\section{NEW PHYSICS UPPER BOUND ON $B\left(B_{s} \rightarrow l^{+} l^{-} \gamma\right)$.}

We repeated the exercise for $B_{s} \rightarrow l^{+} l^{-} \gamma[\underline{5}]$. The radiative decay $B_{s} \rightarrow l^{+} l^{-} \gamma$ is free from helicity suppression due to emission of a photon in addition to the lepton pair. Thus the branching ratio for this leptonic radiative mode is much higher than that for the purely leptonic mode despite an additional factor of $\alpha$. We are interested on how the current data on $b \rightarrow s$ transitions, due to the effective interactions $b \rightarrow s l^{+} l^{-}$and $b \rightarrow s \gamma$, constrain the new physics contribution to the leptonic radiative decays $B_{s} \rightarrow l^{+} l^{-} \gamma$.

Unlike in the case of $B_{s} \rightarrow l^{+} l^{-}$, if new physics is in the form scalar/pseudoscalar, then it makes no contribution to $B_{s} \rightarrow l^{+} l^{-} \gamma$. The photon has $J=1$. Hence the $l^{+} l^{-}$pair also must be in $J=1$ state so that the angular momentum of the final state can be zero. However, by Wigner-Eckert theorem, the matrix element $\left\langle l^{+} l^{-}(J=1)\right| \bar{l}\left(g_{s}+\right.$ $\left.g_{p} \gamma_{5}\right) l|0\rangle$ is zero.

A legitimate question to ask at this stage is: Is it possible to have an order of magnitude or more enhancement of $B_{s} \rightarrow l^{+} l^{-} \gamma$ for any type of new physics operators?

We found that if new physics is in the form of vector/axial-vector operators then the present data on $B \rightarrow\left(K, K^{*}\right) l^{+} l^{-}$doesn't allow a large boost for $B\left(B_{s} \rightarrow l^{+} l^{-} \gamma\right)$. If new phsyics is in the form of tensor/pseudotensor operators, then the data on $B \rightarrow\left(K, K^{*}\right) l^{+} l^{-}$gives no useful constraint but the data on $B \rightarrow K^{*} \gamma$ does. Here again, a large enhancement of $B\left(B_{s} \rightarrow l^{+} l^{-} \gamma\right)$, much beyond the SM expectation, is not possible.

Hence we conclude that the present data on $b \rightarrow$ $s$ transitions allow a large boost in $B\left(B_{s} \rightarrow l^{+} l^{-}\right)$ but not in $B\left(B_{s} \rightarrow l^{+} l^{-} \gamma\right)$, compared to $S M$ expectation.

\section{CONCLUSIONS}

The quark level interaction $b \rightarrow s l^{+} l^{-}$is responsible for the three types of decays (a) semileptonic $B \rightarrow\left(K, K^{*}\right) l^{+} l^{-}$, (b) purely leptonic $B_{s} \rightarrow l^{+} l^{-}$and also (c) leptonic radiative $B_{s} \rightarrow$ $l^{+} l^{-} \gamma$. If $B\left(B_{s} \rightarrow l^{+} l^{-}\right) \geq 10^{-8}$ then the new physics operators responsible for this have to be of the form scalar/pseudoscalar. Such operators have no effect on $B_{s} \rightarrow l^{+} l^{-} \gamma$. Current data on $B \rightarrow\left(K, K^{*}\right) l^{+} l^{-}$and $B \rightarrow K^{*} \gamma$ do not allow any kind of new physics to give rise to a large enhancement of $B\left(B_{s} \rightarrow l^{+} l^{-} \gamma\right)$.

\section{REFERENCES}

1. BaBar Collaboration: B. Aubert et al., Phys. Rev. Lett. 91, 221802 (2003).

2. Belle Collaboration: A. Ishikawa et al., Phys. Rev. Lett. 91, 261601 (2003).

3. A. Ali, E. Lunghi, C. Greub and G. Hiller, Phys. Rev. D 66, 034002 (2002).

4. A. K. Alok, S. Uma Sankar, Phys. Lett. B 620, 61 (2005).

5. A. K. Alok, S. Uma Sankar, hep-ph/0603262

6. A. J. Buras, hep-ph/0101336 and Phys. Lett. B 566, 115 (2003).

7. M. Gronau and D. London, Phys. Rev. D 55, 2845 (1997).

8. J. L. Hewett, S. Nandi and T. G. Rizzo, Phys. Rev. D 39, 250 (1989). H. E. Logan and U. Nierste, Nucl. Phys. B 566, 39 (2000).

9. Diego Tonelli for the CDF Collaboration, hep-ex/0605038.

10. A. Ali, P. Ball, L.T. Handoko and G.Hiller, Phys. Rev. D 61, 074024 (2000). 